Hommand, K. H.

Math. Annalen 140, $442(1960)$

\title{
Berichtigung
}

zu der in Band 140, Seite 22-32 (1960) erschienenen Arbeit

\section{Lokalkompakte zusammenhängende topologische Halbgruppen mit dichter Untergruppe}

Von

KarL Heinrich Hofmann in Tübingen

Die Voraussetzung c) des Hauptsatzes in [2], S. 22-23 muß lauten:

c) Ist 1 das Neutralelement einer dichten offenen Untergruppe, so bildet die $M e n g e$ aller Elemente $x$ aus $K$, die bezüglich 1 kein Inverses besitzen (zu denen also kein $x^{\prime} \in K$ mit $x x^{\prime}=1$ existiert), einen nich t leeren total unzusammenhängenden Unterraum. (Diese Forderung hat ziemlich unmittelbar zur Folge, daß genau ein Element ohne Inverses existiert.)

Anmerkung. Ohne die zwar in [1], aber nicht in [2] nicht ausdrücklich erwähnte Voraussetzung, daß der total unzusammenhängende Unterraum aller Nichtinversen bezüglich 1 wenigstens einen Punkt enthalte, erfüllt jede lokalkompakte, zusammenhängende topologisehe Gruppe die übrigen Voraussetzungen des Satzes, und neben den in der Folgerung des Hauptsatzes genannten drei echten Halbgruppen sind diese topologischen Gruppen die sämtlichen Halbgruppen, die dann den in [2] angegebenen Voraussetzungen des Hauptsatzes genügen. Von der Existenz wenigstens eines Nichtinversen wird in [2], Hilfssatz 2, S. 24 Gebrauch gemacht.

Ich danke Herm Prof. B. L. vaN DER WAERDEN, Zürich, für seinen freundlichen Hinweis auf die Fehlerhaftigkeit der Formulierung des Hauptsatzes in [2].

\section{Litoratur}

[1] Hormann, K. H.: Topologische Doppelloops und topologische Halbgruppen. Math. Ann. 188, 239-258 (1959).

[2] HormaNN, K. H.: Lokalkompakte zusammenhängende topologische Halbgruppen mit diohter Untergruppe. Math, Ann. 140, 22-32 (1960). 